\title{
MODULE OF ANNULUS
}

\section{TOHRU AKAZA and TADASHI KURODA}

1. Let $C$ and $C^{\prime}$ be two simple closed curves in the complex $z$-plane which have no point in common and surround the origin. Denote by $D$ the annulus bounded by $C$ and $C^{\prime}$. Consider a family $\{\gamma\}$ of rectifiable curves $\gamma$ in $D$ and the family $P$ of all non-negative lower semi-continuous functions $\rho=\rho(z)$ in $D$. Put

$$
L_{p}\{r\}=\inf _{r \in[r]} \int_{r} \rho|d z|
$$

Understanding $\frac{0}{0}=\frac{\infty}{\infty}=0$, we call the quantity

$$
\lambda\{r\}=\sup _{\rho \in P} \frac{\left(L_{p}\{r\}\right)^{2}}{\iint_{D} \rho^{2} d \sigma}
$$

the extremal length of the family $\{r\}$, where $d \sigma$ denotes the $\left\{r^{\prime}\right\}$ be the family of all rectifiable curves $r^{\prime}$ in $D$ joining $C$ with $C^{\prime}$ and let $\left\{\gamma^{\prime \prime}\right\}$ be that of all rectifiable curves $\gamma^{\prime \prime}$ in $D$ separating $C$ from $C^{\prime}$. Then it is known that

$$
\lambda\left\{\gamma^{\prime}\right\}=\frac{1}{\lambda\left\{r^{\prime \prime}\right\}}
$$

and that the quantity

$$
\mu=2 \pi \lambda\left\{\gamma^{\prime}\right\}
$$

is the module of $D$. In this note, we give some estimates of $\mu$.

2. Let $D$ be an annulus stated in $\S 1$. We denote by $l_{0}$ the intersection of the half straight line arg $z=\theta(0 \leqq \theta \leqq 2 \pi)$ with $D$ and by $l(\theta)$ the logarithmic length of $l_{0}$, that is,

$$
l(\theta)=\int_{l_{0}} \frac{d r}{r}, \quad z=r e^{i \theta}
$$

Received October 17, 1960. 
The following was proved by Rengel [3].

TheоRem. The module $\mu$ of $D$ satisfies the inequality

$$
\mu \leqq \frac{1}{2 \pi} \int_{0}^{2 \pi} l(\theta) d \theta
$$

Now we shall prove the following which implies Rengel's theorem stated above.

Theorem 1. For the module $\mu$ of $D$, the inequality

$$
\mu \leqq-\frac{2 \pi}{\int_{0}^{2 \pi} \frac{d \theta}{l(\bar{\theta})}}
$$

holds.

Proof. Let $\left\{r^{\prime}\right\}$ be the family of all rectifiable curves $r^{\prime}$ joining $C$ with $C^{\prime}$ in $D$. Then it is obvious that

$$
L_{P}\left\{r^{\prime}\right\} \leqq \int_{l_{\theta}} \rho d r
$$

for any $\rho \in P$ and for any $\theta(0 \leqq \theta \leqq 2 \pi)$. By the Schwarz inequality, we have

$$
\begin{aligned}
\left(L_{\rho}\left\{r^{\prime}\right\}\right)^{2} & \leqq\left(\int_{l_{\theta}} \rho d r\right)^{2} \\
& \leqq \int_{l_{\theta}} d r \int_{l_{\theta}} \rho^{2} r d r=l(\theta) \int_{l_{\theta}} \rho^{2} r d r,
\end{aligned}
$$

or

$$
\left(L_{p}\left\{r^{\prime}\right\}\right)^{2} \frac{1}{l(\theta)} \leqq \int_{l_{0}} \rho^{2} r d r
$$

Integrating both sides with respect to $\theta$, we get

$$
\frac{\left(L_{\mathrm{p}}\left\{\gamma^{\prime}\right\}\right)^{2}}{\iint_{D} \rho^{2} d \sigma} \leqq \frac{1}{\int_{0}^{2 \pi} \frac{d \theta}{l(\theta)}},
$$

which gives

$$
\lambda\left\{\gamma^{\prime}\right\} \leqq \frac{1}{\int_{0}^{2 \pi} \frac{d \theta}{l(\theta)}}
$$

From (2), we obtain our theorem. 
Remark. The Schwarz inequality yields

$$
(2 \pi)^{2}=\left(\int_{0}^{2 \pi} d \theta\right)^{2} \leqq \int_{0}^{2 \pi} \frac{d \theta}{l(\theta)} \int_{0}^{2 \pi} l(\theta) d \theta
$$

from which Rengel's theorem is obtained immediately by using Theorem 1 . In (5), the equality holds if and only if $l(\theta)$ is a constant. In this case, the curve $C^{\prime}$ is obtained as a set of points $\alpha z(z \in C)$, where $\alpha$ is a positive constant, and Rengel's inequality (3) and ours (4) are identical.

3. Here we give an estimate, from below, of the module of an annulus of a special type.

Let $C$ be a simple closed curve in the $z$-plane surrounding the origin and let $(C)$ be a domain bounded by $C$ and containing the origin. If, for any point $z \in C$ and for any $t(0 \leqq t<1)$, the point $t z$ lies in $(C)$, then we say that $C$ is strictly star-like with respect to the origin.

Consider a curve $C$ strictly star-like with respect to the origin. We assume that $C$ consists of a finite number of $\operatorname{arcs} C^{k}: r=r_{k}(\theta), \theta_{k-1} \leqq \theta \leqq \theta_{k}(k=1,2$, $\ldots, n)$, where $\theta_{0}=0, \theta_{n}=2 \pi$ and each $r_{k}(\theta)$ has a continuous derivative $r_{k}^{\prime}(\theta)$ in $\theta_{k-1} \leqq \theta \leqq \theta_{k}$. The expression $r=r(\theta)(0 \leqq \theta \leqq 2 \pi)$ defined by putting $r(\theta)=r_{k}(\theta)$ for $\theta_{k-1} \leqq \theta \leqq \theta_{k}$ is a representation of $C$ in the polar form. In such a case, we say that the curve $C$ is piecewise smooth.

Denote by $C^{\prime}$ the curve defined by $r=\alpha r(\theta)$, where $\alpha$ is a real constant such that $0<\alpha<1$.

We can prove

TheOREM 2. Let $C$ and $C^{\prime}$ be defined as above. Then the module $\mu$ of the annulus $D$ bounded by $C$ and $C^{\prime}$ is estimated from below as follows:

$$
\mu \geqq \frac{1}{K(C)} \log \frac{1}{\alpha},
$$

where $K(C)=\frac{1}{2 \pi} \int_{0}^{2 \pi}\left\{1+\left(\frac{r^{\prime}(\theta)}{r(\theta)}\right)^{2}\right\} d \theta$ is a constant depending only on the curve $C$.

Proof. Let $C_{t}(0 \leqq t \leqq 1)$ be a curve having a representation in the polar form

$$
C_{t}: r=R(\theta, t)=r(\theta)\{\alpha+(1-\alpha) t\}
$$


The curves $C_{1}$ and $C_{0}$ are identical with $C$ and $C^{\prime}$ respectively. Consider the family $\left\{r^{\prime \prime}\right\}$ of all rectifiable curves $r^{\prime \prime}$ separating $C$ from $C^{\prime}$ in $D$ and the family $P$ in $\S 1$. It is easy to see that

$$
\begin{aligned}
d s & =\sqrt{(R(\theta, t))^{2}+\left(\frac{\partial R(\theta, t)}{\partial \theta}\right)^{2}} \\
& =r(\theta)\{\alpha+(1-\alpha) t\} \sqrt{1+\left(\frac{r^{\prime}(\theta)}{r(\theta)}\right)^{2}} d \theta
\end{aligned}
$$

is the line-element along $C_{t}(0<t<1)$ and that

$$
d \sigma=(r(\theta))^{2}(1-\alpha)\{\alpha+(1-\alpha) t\} d \theta d t
$$

is the area-element. It is evident that

$$
L_{p}\left\{\gamma^{\prime \prime}\right\} \leqq \int_{c_{t}} \rho d s
$$

for $0<t<1$. Hence, by the Schwarz inequality, we have

$$
\begin{aligned}
\left(L_{p}\left\{\gamma^{\prime \prime}\right\}\right)^{2} & \leqq\left(\int_{C_{t}} \rho d s\right)^{2} \\
& \leqq \frac{\alpha+(1-\alpha) t}{1-\alpha} \int_{0}^{2 \pi}\left\{1+\left(\frac{r^{\prime}(\theta)}{r(\theta)}\right)^{2}\right\} d \int_{0}^{2 \pi} \rho^{2}(r(\theta))^{2}(1-\alpha)\{\alpha+(1-\alpha) t\} d \theta .
\end{aligned}
$$

Therefore, using the same argument as in the proof of Theorem 1 and noting (1) and (2), we get

$$
\frac{\left(L_{p}\left\{\gamma^{\prime \prime}\right\}\right)^{2}}{\iint_{D} \rho^{2} d \sigma} \leqq K_{0}(C) \frac{1}{\log \frac{1}{\alpha}},
$$

where $K_{0}(C)=\int_{0}^{2 \pi}\left\{1+\left(\frac{r^{\prime}(\theta)}{r(\theta)}\right)^{2}\right\} d \theta$. Putting $K(C)=\frac{1}{2 \pi} K_{0}(C)$, we have our theorem.

EXAMPLE. Let $\Pi_{n}$ be a regular polygon of center at the origin and with $n$ sides of equal length and let $\Pi_{n}^{\prime}$ be another regular polygon obtained from $\Pi_{n}$ by a transformation $z=\alpha z^{\prime}\left(z^{\prime} \in \Pi_{n}\right)$, where $0<\alpha<1$. If we denote by $\mu$ the module of the annulus bounded by $\Pi_{n}$ and $\Pi_{n}^{\prime}$, then, using Theorems 1 and 2 , we get

$$
\frac{1}{K\left(\Pi_{n}\right)} \log \frac{1}{\alpha} \leqq \mu \leqq \log \frac{1}{\alpha}
$$

where 


$$
K\left(\Pi_{n}\right)=\frac{n}{\pi} \tan \frac{\pi}{n}
$$

4. Applying Theorem 2, we prove the following

THEOREM 3. Let $\Delta$ be a domain in the z-plane whose boundary consists of the origin and of an enumerable number of sets $E_{k}(k=1,2, \ldots)$, where $E_{k}$ lies on a simple closed curve $C^{k}$ strictly star-like with respect to the origin and may consist of arcs and points. If there exists a simple closed curve $C$ which is piecewise smooth in the sense stated in \$ 3 and surrounds the origin and if, for each $k$, there exists a positive number $\alpha_{k}$ such that the set of points $\alpha_{k} z$ $(z \in C)$ is contained in $C_{k}$ and such that $\alpha_{k}>\alpha_{k+1}, \lim _{k \rightarrow \infty} \alpha_{k}=0$, then the origin is a weak boundary component of $\Delta$.

Proof. Let us denote by $D_{k}$ the annulus bounded by $C^{k}$ and $C^{k+1}$. Then $D_{k}$ is contained in $\Delta$. Denoting by $\mu_{k}$ the module of $D_{k}$, we see by. Theorem 2 that there exists a constant $K(C)$ depending only on $C$ such that

$$
\mu_{k} \geqq \frac{1}{K(C)} \log \frac{\alpha_{k}}{\alpha_{k+1}} .
$$

Hence we get

$$
\sum_{k=1}^{\infty} \mu_{k} \geqq \frac{1}{K(C)} \sum_{k=1}^{\infty} \log \frac{\alpha_{k}}{\alpha_{k+1}},
$$

whose right hand side diverges. By Grötzsch's theorem [2] (Cf. Savage [4]), we have our assertion.

Remark. This theorem implies Theorem 1 in [1].

\section{References}

[1] T. Akaza: On the weakness of some boundary component, Nagoya Math. Journ., 17 (1960), 219-223.

[2] H. Grötzsch: Eine Bemerkung zum Koebeschen Kreisnormierungsprinzip, Berichte Verl. Sächs. Akad. Wiss. Leipzig Math. Nat., K1. 87 (1935), 319-324.

[3] E. Rengel: Über einige Schlichttheoreme der konformer Abbildung, Schrif. d. Math. Sem. u. Inst. f. angew. Math., Univ. Berlin, 1 (1932-33), 141-162.

[4] N. Savage: Weak boundary components of an open Riemann surface, Duke Math. Journ., 24 (1957), 79-95.

Kanazawa University

and

Nagoya University 